\title{
Research on vehicle speed detection technology based on micro-magnetoresistive sensing equipment
}

\author{
Wei Zhang, Li Wang*, Chunli Li, Mi Chen, and Sheng Tao \\ China Academy of Transportation Sciences, Beijing 100029, China
}

\begin{abstract}
In order to effectively detect road traffic to achieve intelligent road traffic management, the vehicle speed detection algorithm based on micro-magnetoresistive sensing equipment was studied. A new pairwise differential eigenvalue vehicle speed calculation algorithm was proposed to solve the problem that selecting one eigenvalue on the magnetoresistive curve may be influenced by the actual condition that the data curves of the two sensors and the relative base voltage cannot be exactly the same. The results of the application on a certain expressway indicate that speed detection accuracy can be $95.4 \%$, besides, both the integrity and the validity of the data can meet the requirements.
\end{abstract}

Key words. Road engineering; Vehicle speed detection; Pairwise differential eigenvalues; Micromagnetoresistive sensor

\section{Introduction}

Real-time road traffic information collection is the basis of road administration department to make road monitoring management, traffic information analysis and relevant research and decision. Enhancing research on traffic information sensing technology and equipment is the premise to achieve intelligent road traffic management.

As a kind of shallow-embedded vehicle detection equipment, micro-magnetoresistive vehicle detector has the characteristic such as no reaction to nonferromagnetic objects, informative, small and light and easy to be installed. It has outstanding effect in the application of dynamic and static vehicle detection, estimate of vehicle speed and length as well as classification of vehicle type $\mathrm{e}^{[1,2,3,4]}$.

In recent years, many relevant scientific research institutes and enterprises at home and abroad have been conducting research on magnetoresistive vehicle detection technology. How to separate vehicle information from continuous signal sequence is the problem that needs to be solved by magnetoresistive sensor vehicle recognition algorithm. Sing ${ }^{[5]}$ introduced a fixed threshold value detection algorithm, but it doesn't solve the problem that the sensor is liable to have datum drift and the distortion. In order to address the defect of threshold value judgment, Knaian ${ }^{[6]}$ proposed state machine detection method, which does not only judge the existing state of vehicles, but also judges the information such as coming, existing and leaving of vehicles. The subdivision of the state enhances the robustness of detection algorithm and improves algorithm recognition rate, but the drift of datum value of sensor still exists. In a word, detection algorithm is mainly affected by noise, discontinuity of detection signal and the drift of datum value. At present, there is no good solution for drift of datum value and the commonly used method is re-calibration of datum value regularly.

This paper conducted research on vehicle speed detection algorithm based on micro-magnetoresistive sensing equipment and proposed a pairwise differential eigenvalue vehicle speed calculation algorithm, which reduced the error of vehicle speed detection caused by drift of datum value and effectively improved the vehicle speed detection accuracy of micromagnetoresistive sensing equipment.

\section{Research on vehicle speed detection algorithm based on micro- magnetoresistive sensing equipment}




\subsection{Vehicle speed detection principle}

The principle of vehicle speed detection with micromagnetoresistive sensing equipment is: when a vehicle comes close to magnetoresistive equipment, signal of magnetoresistive 1 and 2 will exceed threshold value successively and produce similar changing Curve. As shown in Figure 1, under ideal circumstance, signal curve of magnetoresistance 2 could overlap with that of magnetoresistance 1 when making time-domain translation $\Delta t$, that is, a corresponding similar point $A_{2}$ could be found on magnetoresistance 2 if $A_{1}$ is taken randomly on the curve of magnetoresistance 1 . The time difference between the two points is the time delay $\Delta t$ required for calculating speed. Thus, as long as the accurate time delay is got, the accuracy of vehicle speed calculation can be ensured. Supposing serial number of sampling point of $A_{1}$ is $N_{1}$ while that of sampling point of $A_{2}$ is $N_{2}$, sampling frequency is $f_{\mathrm{sp}}$ and the distance between two sensors is $l$, vehicle speed could be expressed as:

$$
v=l \cdot f_{s p} /\left(N_{2}-N_{1}\right)
$$

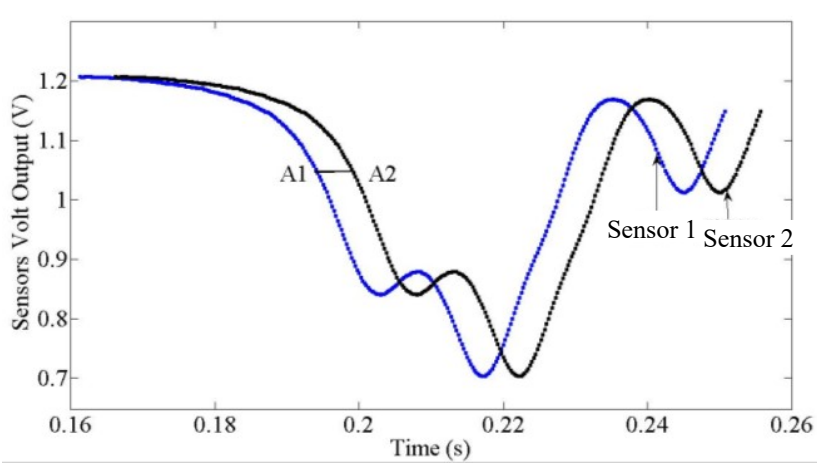

Fig.1. Schematic of Speed Detection

However, as the static output voltage of the two magnetoresistive sensors is different in practical application, there is certain difference between the magnification times of signal in module. Besides, in case the driving direction of vehicle has certain included angle with axial direction of magnetoresistive equipment, the part of automobile passing two magnetoresistances will also differ, which, combining with random jitter of vehicle and road roughness, will result in different signal sensed by the two sensors. Therefore, under the influence of many factors, the curve of signal sensed by two magnetoresistances cannot completely overlap in practice. Thus, we cannot select two similar points on magnetoresistive curve randomly, but shall select appropriate eigenvalue to calculate time delay. The research and practical result indicates selecting the peak value of the two magnetoresistive curves or the threshold value triggered by the coming of vehicles as eigenvalue to calculate time delay could guarantee the accuracy of time delay calculation.

\subsection{Vehicle detection error caused by signal amplitude difference}

It is found in practical application that the data curves of the two sensors and the relative base voltage cannot be exactly the same, thus calculation with one eigenvalue may not get better effect. As shown by measurement signal in Figure 2, Figure 2 (b) is the local amplification of Figure 2 (a).

In Figure 2, two signals have been subtracted with base signal. After careful observation of Figure 2(b), it can be found although the two curves have similar shape, their amplitude varies, which result in: in case single eigenvalue of one time is used to calculate speed, there will be speed measurement error as the signal amplitude of the two sensors is different.
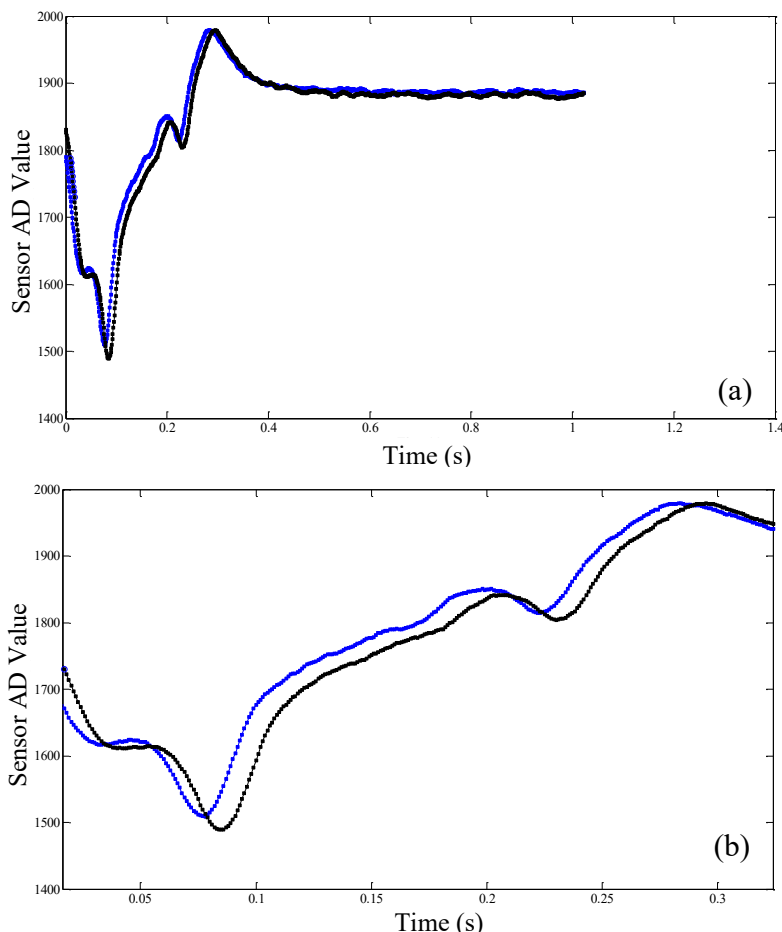

Fig.2. Filtered Signal of Magnetoresistive Sensor (a) global curve (b) local magnified curve

The principle of the measurement error caused by signal amplitude differences is shown in Figure 3, obviously, $t_{2}>t_{1}$ in the figure, which means the speed calculated with difference between two times has error. Supposing actual time difference is $t_{0}, t_{l}$ could be expressed as $t_{1}=t_{0}-d t$ while $t_{2}$ as $t_{2}=t_{0}+d t$.

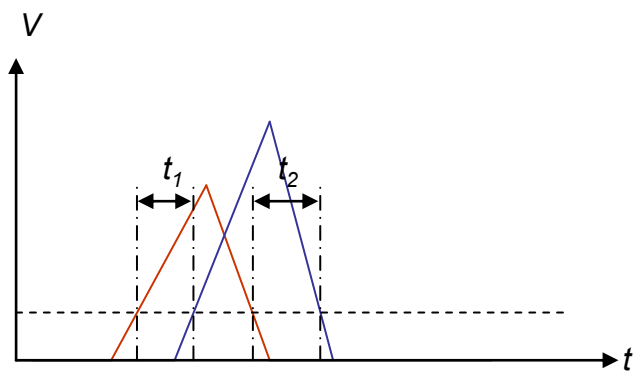

Fig.3. Schematic Diagram of Speed Measurement Error Caused by Signal Amplitude Differences 


\subsection{Vehicle speed detection algorithm based on pairwise differential eigenvalue}

In order to solve the problem of vehicle speed detection error caused by signal amplitude difference, previous method is to make signal normalization processing by using hardware or software, but this method needs to do lots of test and the consistency of signal value cannot be absolutely guaranteed. It can be seen from aforesaid analysis that the average value of $t_{2}$ and $t_{l}$ is true value $t_{0}$. Based on this method, this paper proposed a kind of method of calculating average time with pairwise differential eigenvalue. Its schematic diagram is described as follows.

First of all, set threshold value $T$ and difference value $d$, and then make binarization processing of sensor signal $S$. The method is as shown in formula (2).

$$
S_{n}^{\prime}=\left\{\begin{array}{l}
1, S>(T-d), S_{n-1}^{\prime}=1 \\
1, S>(T+d), S_{n-1}^{\prime}=0 \\
0, S<(T+d), S_{n-1}^{\prime}=0 \\
0, S<(T-d), S_{n-1}^{\prime}=1
\end{array}, S_{0}^{\prime}=0\right.
$$

Where, $T-d$ and $T+d$ are referred to as a pair of pairwise differential eigenvalue.

At last, make XOR of binarization sequence signal of the two sensors to get time difference sequence $\left\{T_{d}\right\}$, and take even-even time difference from the sequence, calculate its average value and use it as actual vehicle passing time $t$ to calculate final speed.

This method could effectively reduce the speed measurement error caused by the difference of sensor signal amplitude. In addition, the hysteresis effect caused by $2 d$ could effectively reduce the influence of fluctuation of signal in a small range.

\section{Installation of micro- magnetoresistive vehicle detection equipment}

In Figure 4, equipment arrangement diagram is provided with a unidirectional three-lane highway as an example. The arrangement method of bidirectional multi-lane is similar. Magnetoresistive equipment is a cylindrical one and single magnetoresistive equipment also has directionality, which is referred to as axial direction of equipment in this research. The arrow in the figure represents the driving direction, that is, the direction from magnetoresistance 1 to 2 within the equipment. It means when a vehicle arrives, it will be sensed by magnetoresistance 1 and 2 successively.

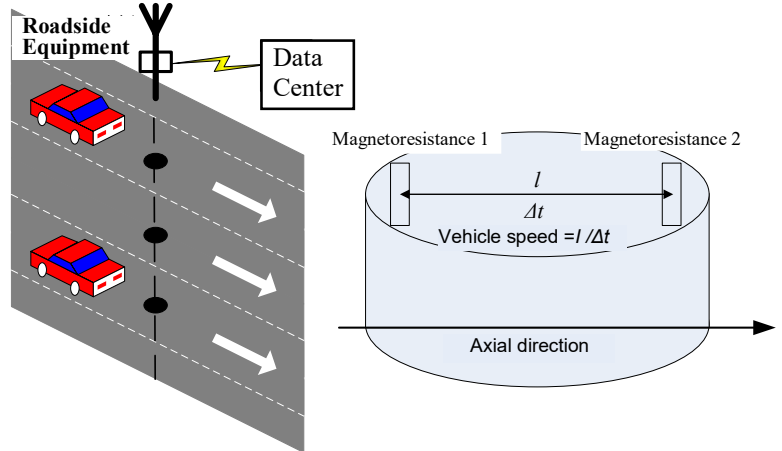

Fig .4. General Scheme of Magnetoresistive Equipment

Magnetoresistive sensing equipment is installed at the center of every lane of the road and is kept to be at a straight-line vertical to the driving direction. One magnetoresistive equipment can only be used to detect vehicles passing such lane. By adjustment of threshold value, the magnetoresistive equipment located at the center of some lane can only be triggered by the vehicle passing right over or above, thus there is no interference from adjacent lanes.

In case magnetoresistive equipment is buried under the pavement, the plane of its top shall be parallel to and be slightly lower than the pavement, and be sealed and covered with proper road repair material. In case it is installed on pavement, the axial direction of magnetoresistive equipment shall be parallel to the driving direction of vehicles and the arrow shall point to driving direction. In view of the microminiaturization of magnetoresistive equipment, the pavement drilling diameter shall be about $150 \mathrm{~mm}$. Thus, the distance between two magnetoresistive sensors shall be set as $l=120 \mathrm{~mm}$. The vehicle speed $v=$ $l / \Delta t$ can be calculated by measuring the time delay of sensing of vehicles by the two magnetoresistive equipment.

\section{Application of micro- magnetoresistive vehicle detection equipment}

In order to inspect the practical application effect, 54 sets of magnetoresistive sensing equipment and 14 sets of roadside antenna receiving equipment are installed at certain expressway, which can provide average speed and flow of traffic stream at 14 cross sections. Figure 5 is the actual installation picture of micromagnetoresistive vehicle detection equipment on an interchangeable cross section. This cross section is composed of two lanes and every lane is installed with micro- magnetoresistive vehicle detection equipment in the center. 


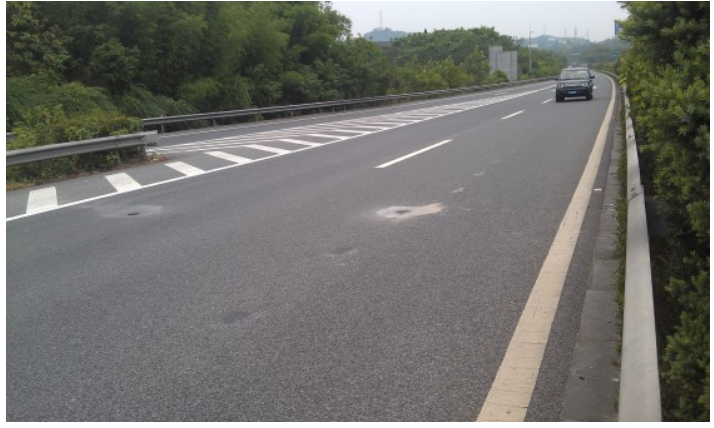

Fig.5. Installation Diagram of Expressway Interchangeable Magnetoresistive

In order to verify the influence of pairwise differential eigenvalue on vehicle speed detection accuracy proposed, several traffic cross sections installed with micro-magnetoresistive vehicle detection equipment are randomly selected as test points and hand-held microwave vehicle speed detector with detection accuracy as $1 \%$ are used to detect and record the speed of vehicles passing such cross section, which is used to compare with the vehicle speed measured by magnetoresistive vehicle detector. The number of sample vehicle detected is 100 and the speed range of test sample is $20 \sim 120 \mathrm{~km} / \mathrm{h}$. The two algorithms used in verification could be downloaded to vehicle detection equipment with wireless communication method.

First of all, the simple eigenvalue vehicle detection algorithm with peak eigenvalue is tested. Through test and sample arrangement calculation, $89.1 \%$ expectation of vehicle speed detection is got.

Secondly, vehicle detection algorithm with pairwise differential eigenvalue is tested. Through test and sample arrangement calculation, 95.4\% expectation of vehicle speed detection is got. Vehicle speed detection accuracy is greatly improved.

\section{Conclusion}

This paper mainly conducted research on vehicle speed detection algorithm of micro-magnetoresistive vehicle detection equipment and developed a kind of magnetoresistive traffic flow collection equipment with low power consumption and small size based on the algorithm which s relatively higher practical value.

In the research of vehicle speed measurement algorithm, the pairwise differential eigenvalue vehicle speed calculation algorithm is created with the aim of reducing the defect of existing algorithm in practical application, and the speed measurement error caused by the difference of signal amplitude of sensor is calibrated. The result of application on a certain expressway shows the vehicle speed detection accuracy of this equipment is above 95\%. This research creates condition for building road traffic flow monitoring system.

\section{References}

1. S. Y. Cheung, S. C. Ergen, and P. Varaiya, "Traffic Surveillance with Wireless Magnetic Sensors", ITS World Congress, 2005:648-649.

2. M. H. Kang, B. W. Choi, K. C. Koh, et al., Experimental study of a vehicle detector with an AMR sensor, Sensors \& Actuators A Physical, 2005, 118(2):278-284.

3. M. J. Caruso, C. H. Smith, T. Bratland, et al., A New Perspective on Magnetic Field Sensing, Sensors, 1998, 15(12).

4. X. Li, G. Yu, Application of AMR sensors to vehicle detection, Journal of University of Science and Technology Beijing, 2006, 28(6):587-590.

5. S. Y. Cheung, S. Coleri, B. Dundar, et al., Traffic measurement and vehicle classification with single magnetic sensor, Transportation Research Record, 2005, 10.

6. A. N. Knaian, J. Paradiso, A. C. Smith, A Wireless Sensor Network for Smart Roadbeds and Intelligent Transportation Systems, Massachusetts Institute of Technology, 2000. 